\title{
Biosynthesis of agar in red seaweeds: a review
}

\begin{abstract}
Agar is a jelly-like biopolymer synthesized by many red seaweeds as their major cell wall component. Due to its excellent rheological properties, it has been exploited commercially for applications in food, cosmetic, pharmaceutical, biomedical and biotechnology industries. Despite its multiple uses, the biosynthesis of this phycocolloid is not fully understood. The current knowledge on agar biosynthesis is inferred from plant biochemistry and putative pathways for ulvan and alginate biosynthesis in green and brown seaweeds, respectively. In this review, the gaps in our current knowledge on agar biosynthetic pathway are discussed, focusing on the biosynthesis of agar precursors, elongation of agar polysaccharide chain and side chain modification. The development of molecular markers for the screening of desired seaweeds for industrial exploitation is also discussed.
\end{abstract}

Keyword: Agar; Agar biosynthesis; Phycocolloid; Rhodophyta; Seaweed 